\title{
Potentially inappropriate medications in older patients based on Beers criteria: a cross-sectional study of a family medicine practice in Saudi Arabia
}

\begin{abstract}
Atheer Alturki, MD 1 , Tareef Alaama, FACP, FRCPC 2,3, Yousef Alomran, MD4, Ahmed Al-Jedai, MBA, PharmD, BCPS, FCCP, FAST ${ }^{5,6}$, Hajer Almudaiheem, MSc, PharmD7, Ghassan Watfa, MD, PhD ${ }^{8 *}$
\end{abstract}

${ }^{1}$ Family Medicine Specialist, King Saud Medical City, Central Health Cluster One, Riyadh, Kingdom of Saudi Arabia; ${ }^{2}$ Deputy Minister for Therapeutic Affairs, Ministry of Health, Deputyship of Therapeutic Affairs, Riyadh, Kingdom of Saudi Arabia; ${ }^{3}$ Assistant Professor and Consultant of Internal Medicine \& Geriatric Medicine, King Abdulaziz University, Jeddah, Kingdom of Saudi Arabia; ${ }^{4}$ Consultant of Family Medicine and Associate Executive Director for Primary Care and Community Health, King Saud Medical City, Central Health Cluster One, Riyadh, Kingdom of Saudi Arabia; ${ }^{5}$ Assistant Deputy Minister for Medical Support Services, Ministry of Health, Deputyship of Therapeutic Affairs, Riyadh, Kingdom of Saudi Arabia; ${ }^{6}$ Professor and Consultant Clinical Pharmacist, Solid Organ Transplant, College of Medicine and Pharmacy, Alfaisal University, Riyadh, Kingdom of Saudi Arabia; ${ }^{7}$ Drug Policy Department Manager, Ministry of Health, Deputyship of Therapeutic Affairs, Riyadh, Kingdom of Saudi Arabia; ${ }^{8}$ Consultant of Geriatric Medicine and Director of Home Health Care Administration, King Saud Medical City, Central Health Cluster One, Riyadh, Kingdom of Saudi Arabia

*For correspondence: g.watfa@ ksmc.med.sa

Competing interest: The authors declare that no competing interests exist.

Received: 31 July 2019

Accepted: 03 October 2019

Published: 05 February 2020

(C)This article is Open Access: CC BY license (https://creativecommons.org/licenses/ by/4.0/)

Author Keywords: Saudi Arabia, family medicine, potentially inappropriate medications, Beers criteria, geriatrics, aged

Copyright (C) 2020, The Authors; DOI:10.3399/

bjgpopen20X101009

\section{Abstract}

Background: The use of potentially inappropriate medications (PIMs) is an important issue in older patients who are at risk of adverse drug events.

Aim: To determine the prevalence of PIM use, according to Beers criteria, among an older population (aged $\geq 65$ years) in a large family medicine setting, and to identify the associated risks.

Design \& setting: A prospective cross-sectional study of patients aged $\geq 65$ years was conducted from June 2017 to June 2018 at the Family and Community Medicine (FCM) clinics of King Saud Medical City (KSMC) in Riyadh, Saudi Arabia.

Method: This cross-sectional study included patients aged $\geq 65$ years who were seen at new appointments or followed-up at the FCM clinics of KSMC in Riyadh, Saudi Arabia. Data were collected by extensive face-to-face interviews and from the patients' medical records.

Results: A total of 270 older patients aged $72.41 \pm 6.23$ years (mean \pm standard deviation [SD]) were included in the present study. The prevalence of PIMs was $60.7 \%(n=164)$. Multivariate analyses identified three independent variables associated with PIMs: incremental age per 5 years (odds ratio [OR] 1.47, 95\% confidence intervals $[\mathrm{Cl}]=1.15$ to $1.88 ; P=0.002)$, female sex $(\mathrm{OR} 1.95,95 \% \mathrm{Cl}=$ 1.10 to $3.42 ; P=0.021)$, and polypharmacy $(\mathrm{OR} 8.21,95 \% \mathrm{Cl}=4.58$ to $14.7 ; P<0.001)$. The most common PIMs used were $39.4 \%$ related to proton pump inhibitors (PPI), $25.2 \%$ to diuretics (other than spironolactone), $10.6 \%$ to non-steroidal anti-inflammatory drugs (NSAIDs), and $8.7 \%$ to aspirin use. 
Conclusion: This study showed high prevalence of PIMs. Increasing age, female sex, and polypharmacy were found to be significant risk factors for PIM use. The frequency of morbidities was not significantly different among patients with PIMs compared to those without PIMs, except for hypertension and osteoarthritis, which were more common in the PIMs group. The present study reinforces the importance of comprehensive medication management and reviews.

\section{How this fits in}

PIM usage is a problem among older patients who are at risk of adverse drug events. This study, conducted in Saudi general practice, showed a highly prevalent use of PIMs $(60.7 \%, n=164)$, which is higher than the rates previously reported in western countries, and even higher than the previous studies from Saudi Arabia. The present study reinforces the importance of conducting comprehensive medication management for older patients in general practice to avoid any potential harm related to PIMs.

\section{Introduction}

Inappropriate medication usage is an issue among older patients (aged $\geq 65$ years), particularly those who have concomitant morbidities. They are more likely to experience drug-related adverse events than younger populations. ${ }^{1,2}$ Drugs are usually given to older patients based on the efficacy and safety studies conducted among younger patients who do not have similar morbidities. ${ }^{3,4}$ Causation of drugrelated adverse events in older patients is multifactorial, including complex medication regimens, ageing, and changes in medicine pharmacokinetics and pharmacodynamics. ${ }^{5}$

It has been reported that the adverse drug events related to PIMs are associated with the rate of hospitalisation. ${ }^{6}$ The use of inappropriate medications in this population has been shown to cause approximately $5 \%$ of hospital admissions. ${ }^{7}$ Moreover, a further study has shown interacting medications leading to adverse drug reactions in up to $35 \%$ of older outpatients, and $40 \%$ of inpatients with inappropriate medication use. ${ }^{8}$ The Beers criteria were developed by Mark $\mathrm{H}$ Beers in 1991 at the University of California, Los Angeles, with subsequent updates. It was adopted later by the American Geriatrics Society, which recently updated the criteria in 2015. It is used to assess medication safety among older patients. ${ }^{9}$ It highlights the medications to be avoided in older patients, as well as the medications that should be avoided in certain clinical conditions or concomitantly with other interacting medications and those to be used with caution in this sensitive age group. ${ }^{10}$ The older patient population is vulnerable to physical impairments and health problems. They are more likely to endure chronic health conditions, deficiencies, digestive problems, impaired metabolism and excretion, and susceptibility to adverse drug effects.

The demographic of the Saudi population is changing. It is a growing population with an increasing life expectancy, and a high prevalence of chronic diseases. In 2015, 3.1\% of the Saudi Arabian population was aged $\geq 65$ years. Projections show that the proportion of the older population (aged $\geq 65$ years) will continue to increase and will eventually reach $6.6 \%$ of the total Saudi population in 2030 and $16.7 \%$ by $2050 .{ }^{11}$

In Canada, a higher proportion of females (42.0\%) than males $(31.0 \%)$ were shown to have filled PIM prescriptions. ${ }^{12}$ Family doctors were found to prescribe sedatives and/or hypnotics more frequently in older patients for many reasons: limited knowledge regarding PIMs; limited applicability of PIMs lists in daily practice; lack of time; and lack of therapeutic alternatives. Other patient-related factors that also affected family doctors' prescribing practice include: bad experiences regarding changes of medication; refusal to follow prescriptions of sedative and/or hypnotics with doses that are not considered PIMs; and refusal to stop the prescribed sedatives and/or hypnotics if necessary. This resulted in some form of resignation on the physician's side. ${ }^{13}$ An Italian study showed a lower percentage of PIM use (28.0\%), and that females were more likely to be exposed to PIMs than males. ${ }^{14}$ In Taiwan, the prevalence of having at least one PIM was reported to be high at $82.67 \%$, and these were mostly prescribed by internist or family physicians and neurologists or psychiatrists. ${ }^{15}$

The present study was conducted to determine the prevalence of PIM use among older patients (aged $\geq 65$ years) in a family medicine setting in Saudi Arabia based on the Beers 2015 criteria, and to identify the factors associated with PIMs. 


\section{Method}

This cross-sectional study was conducted from 1 June 2017-31 May 2018 at the FCM clinics of KSMC, a 1351-bed tertiary care hospital in Riyadh, Saudi Arabia. The FCM clinics are a set of clinics serving a catchment area of multiple primary health care centers (PHCs). The FCM clinics are based in a tertiary care institute and receive patients that are referred from PHCs. Hence, patients seen at the clinics had medical needs that could not be solved at the level of the PHC and needed more resources or capabilities. Family physicians see patients referred from PHCs on an appointment basis as new referrals, and further follow-up appointments are scheduled if needed. The present study included all patients aged $\geq 65$ years who were seen by the family physicians at KSMC FCM clinics with new appointments or as follow-up. Patients who had undergone organ transplantation were excluded from the study due to their inevitably high polypharmacy. Only patients with medical records at KSMC were invited for study participation, as FCM clinics also receive patients outside of the catchment area for whom medications are not well documented.

Non-probability convenience sampling was used to recruit participants. Older patients were invited and informed about the purpose of the study. Written informed consent was collected from all consenting patients and from Healthcare Power of Attorney for patients with severe dementia, or patients who were not capable of making decisions on their own.

Data were collected using a case report form, which included demographic profiles of the patients (age and sex). Clinical data were additionally collected by extensive face-to-face interviews and from the patients' medical records.

During face-to-face interviews, all patients underwent clinical evaluations, providing a verbal medical history to the interviewer, which was subsequently verified against the patients' medical records. The presence of any morbidity was classified into two categories 'Yes' and 'No'. The results of biological analyses were captured from the patients' medical records.

Information on the nature of any currently prescribed drugs was collected from the patients' medical records and classified into two categories, 'Yes' and 'No' (medications were identified by their pharmacological class). The number of drugs taken on a daily basis and long-term basis were also recorded. Polypharmacy was evaluated with a binary parameter as the consumption of $\geq 4$ different drugs chronically on a daily basis. ${ }^{16}$ The American Geriatrics Society 2015 Updated Beers Criteria ${ }^{9}$ were used to determine PIMs use. All collected data were entered on the study case report form.

\section{Statistical analysis}

Sample size was calculated using the Metcalfe formula. ${ }^{17}$ Assuming $52.5 \%$ prevalence of PIMs in older patients, ${ }^{7}$ and with $6 \%$ margin of error, $80 \%$ power, and $95 \% \mathrm{Cl}$, the calculated sample size was 270 patients.

All analyses were carried out using the Statistical Package for Social Sciences (version 23). Univariate analyses were performed after individuals were stratified into two groups according to PIMs use; $\chi^{2}$ tests were performed when comparing categorical variables between groups; and t-tests were used for continuous variables, as the sample distribution is normal. The significance of the results is represented as a $P$ value. A $P$ value of $<0.05$ was considered statistically significant. Results are presented as mean \pm SD for quantitative variables and as a percentage for categorical variables.

Logistic regression model analysis was performed with an interactive backward selection method in order to develop a model including parameters associated with PIM use. Regression coefficients, ORs, and $95 \% \mathrm{Cls}$ were calculated. Validity of the model assumption was verified using analysis of model residuals and testing for heteroscedasticity.

\section{Results}

Among the 282 patients invited to take part in the study, 12 patients did not agree to participate (95.7\% acceptance rate). A total of 270 older patients were included in the study (143 female, 53.0\%; mean age $72.41 \pm 6.23$ years). There were 77 patients $(28.5 \%)$ with one morbidity, 109 (40.4\%) with two morbidities, and 81 (30.0\%) with $>2$ morbidities. Three patients had no comorbidities. There were 164 patients (60.7\%) who reported having used PIMs: three patients had four PIMs (1.8\%); 17 patients had three PIMs (10.4\%); 34 patients had two PIMs (20.7\%); and the remaining 110 patients had only one PIM (67.1\%). 
Table 1 Demographics and frequency of morbidities

\begin{tabular}{|c|c|c|c|c|}
\hline \multirow[b]{2}{*}{ Parameter } & \multirow[b]{2}{*}{ All patients } & \multicolumn{2}{|c|}{ PIM $^{a}$} & \multirow[b]{2}{*}{$P$ value } \\
\hline & & Yes & No & \\
\hline Total & 270 & 164 & 106 & \\
\hline Mean age, years $\pm S D$ & $72.41 \pm 6.23$ & $73.09 \pm 6.68$ & $71.35 \pm 5.33$ & 0.024 \\
\hline Female sex, $n(\%)$ & $143(52.9)$ & 95 (57.9) & $48(45.3)$ & 0.042 \\
\hline Mean number of medications $\pm S D$ & $4.09 \pm 2.19$ & $4.84 \pm 2.09$ & $2.88 \pm 1.76$ & $<0.001$ \\
\hline Polypharmacy, n (\%) & $149(55.2)$ & $119(72.6)$ & $30(28.3)$ & $<0.001$ \\
\hline Mean number of morbidities \pm SD & $2.11 \pm 1.14$ & $2.16 \pm 0.95$ & $2.04 \pm 1.39$ & 0.397 \\
\hline Hypertension, $n(\%)$ & $196(72.6)$ & $130(79.3)$ & $66(62.3)$ & 0.002 \\
\hline Diabetes mellitus, $n(\%)$ & $146(54.1)$ & $91(55.5)$ & $55(51.9)$ & 0.562 \\
\hline Dyslipidaemia, n (\%) & $59(21.9)$ & $38(23.2)$ & $21(19.8)$ & 0.540 \\
\hline Low back pain, $n(\%)$ & $15(5.6)$ & $9(5.5)$ & $6(5.7)$ & 0.952 \\
\hline Heart failure, $n(\%)$ & $11(4.1)$ & $9(5.5)$ & $2(1.9)$ & 0.144 \\
\hline Stroke, n (\%) & $9(3.3)$ & $6(3.7)$ & $3(2.8)$ & 0.711 \\
\hline Chronic kidney disease, n (\%) & $4(1.5)$ & $2(1.2)$ & $2(1.9)$ & 0.658 \\
\hline Diabetic nephropathy, n (\%) & $2(0.7)$ & $1(0.6)$ & $1(0.9)$ & 0.755 \\
\hline Osteoporosis, n (\%) & $20(7.4)$ & $13(7.9)$ & $7(6.6)$ & 0.675 \\
\hline Osteoarthritis, n (\%) & $15(5.6)$ & $13(7.9)$ & $2(1.9)$ & 0.034 \\
\hline Bronchial asthma, n (\%) & $9(3.3)$ & $5(3.0)$ & $4(3.8)$ & 0.735 \\
\hline Hyperuricaemia, n (\%) & $2(0.7)$ & $1(0.6)$ & $1(0.9)$ & 0.755 \\
\hline Hypothyroidism, n (\%) & $21(7.8)$ & $11(6.7)$ & $10(9.4)$ & 0.414 \\
\hline Anaemia, $n(\%)$ & $4(1.5)$ & $2(1.2)$ & $2(1.9)$ & 0.658 \\
\hline $\mathrm{BPH}, \mathrm{n}(\%)$ & $22(8.1)$ & $12(7.3)$ & $10(9.4)$ & 0.535 \\
\hline
\end{tabular}

$\mathrm{BPH}=$ benign prostatic hypertrophy. $\mathrm{PIM}=$ potentially inappropriate medication.

${ }^{\text {aP }}$ atients taking $\geq 1 \mathrm{PIMs}$. ${ }^{\text {PP }}$ robability of the $t$-test (continuous variables) or $\chi^{2}$ tests (categorical variables).

Table 1 shows the demographic variables, and the common chronic diseases of the population study, as well as a comparison between patients with and without PIMs.

The average number of morbidities in the cohort was $2.11 \pm 1.14$ morbidities. The prevalence of hypertension and diabetes was high among participants $(72.6 \%$ and $54.1 \%$, respectively). Polypharmacy was recorded at $25.2 \%$ and was significantly more common among patients with PIMs than those without PIMs. Moreover, patients with PIMs had higher hypertension prevalence than patients without PIMs ( $P=0.002)$, as shown in Table 1. Patients with PIMs were also more frequently treated with beta-blockers, calcium channel blockers (CCBs), and angiotensin-converting enzyme (ACE) inhibitors (Table 2).

Compared to patients without PIMs, patients with PIMs were significantly older $(73.09 \pm 6.68$ years versus $71.35 \pm 5.33$ years; $P=0.024$ ) with a higher proportion of females $(n=95 / 164,57.9 \%$ versus $n=$ $69 / 164,42.1 \% ; P=0.042$ ). The frequency of morbidities was not significantly different among patients with PIMs compared to those without PIMs; however, significant use of PIMs was found in patients with hypertension and osteoarthritis $(P=0.002$ and $P=0.034$, respectively) (Table 2).

There were no documented cases of chronic obstructive pulmonary disease, gastroesophageal reflux disease, vitamin D deficiency, or prostate cancer among patients with PIMs, and only one case of each disease in the patient group without PIMs (data not shown).

The number of medications was significantly higher among patients with PIMs than those without PIMs (4.84 \pm 2.09 versus $2.88 \pm 1.76 ; P<0.001)$, with higher polypharmacy prevalence in the PIMs subgroup compared to those without PIMs (72.6\% and $28.3 \%$, respectively; $P<0.001)$ (Table 1). 
Table 2 Frequency of medications used

\begin{tabular}{|c|c|c|c|c|}
\hline \multirow[b]{2}{*}{ Medication } & \multirow[b]{2}{*}{$\begin{array}{l}\text { All patients, } n(\%) \\
\quad(n=270)\end{array}$} & \multicolumn{2}{|c|}{ PIMs, $n(\%)^{\mathrm{a}}$} & \multirow[b]{2}{*}{$\begin{array}{c}P \text { val- } \\
\text { ue }^{\text {b }}\end{array}$} \\
\hline & & $\begin{array}{l}\text { Yes, } n(\%) \\
(n=164)\end{array}$ & $\begin{array}{l}\text { No, } n(\%) \\
(n=106)\end{array}$ & \\
\hline Metformin & $116(43.0)$ & $74(45.1)$ & $42(39.6)$ & 0.373 \\
\hline Sulfonylureas & $74(27.4)$ & $44(26.8)$ & $30(28.3)$ & 0.791 \\
\hline DPP4 Inhibitors & $28(10.4)$ & $18(11.0)$ & $10(9.4)$ & 0.685 \\
\hline TZDs & $2(0.7)$ & $1(0.6)$ & $1(0.9)$ & 0.755 \\
\hline Beta-blockers & $30(11.1)$ & $24(14.6)$ & $6(5.7)$ & 0.022 \\
\hline $\mathrm{CCBs}$ & $92(34.1)$ & $64(39.0)$ & $28(26.4)$ & 0.033 \\
\hline ACE inhibitors & $67(24.8)$ & $51(31.1)$ & $16(15.1)$ & 0.006 \\
\hline ARBs & $50(18.5)$ & $33(20.1)$ & $17(16.0)$ & 0.399 \\
\hline Clopidogrel & $17(6.3)$ & $13(7.9)$ & $4(3.8)$ & 0.170 \\
\hline PPIs & $94(34.8)$ & $91(55.5)$ & $3(2.8)$ & $<0.001$ \\
\hline NSAIDs & $55(20.4)$ & $35(21.3)$ & 20 (18.9) & 0.622 \\
\hline Aspirin & $112(41.5)$ & $82(50.0)$ & $30(28.3)$ & $<0.001$ \\
\hline Thyroxine & $22(8.1)$ & $11(6.7)$ & $11(10.4)$ & 0.282 \\
\hline Statins & $141(52.2)$ & $97(59.1)$ & $44(41.5)$ & 0.005 \\
\hline Antibiotics & $5(1.9)$ & $2(1.2)$ & $3(2.8)$ & 0.338 \\
\hline Antihistamines & $23(8.5)$ & $20(12.2)$ & $3(2.8)$ & 0.007 \\
\hline Bisphosphonates & $12(4.4)$ & $8(4.9)$ & $4(3.8)$ & 0.667 \\
\hline Teriparatide & $3(1.1)$ & $1(0.6)$ & $2(1.9)$ & 0.328 \\
\hline Tolterodine & $1(0.4)$ & $0(0.0)$ & $1(0.9)$ & 0.213 \\
\hline Tamsulosin & $20(7.4)$ & $10(6.1)$ & $10(9.4)$ & 0.307 \\
\hline Finastride & $11(4.1)$ & $5(3.0)$ & $6(5.7)$ & 0.289 \\
\hline
\end{tabular}

a Patients taking $\geq 1$ PIMs. ${ }^{b}$ Probability of $\chi^{2}$ tests (categorical variables).

$\mathrm{ACE}=$ angiotensin-converting enzyme. $\mathrm{ARBs}=$ angiotensin receptor blockers. $\mathrm{CCBs}=$ calcium channel blockers. DPP4 = dipeptidyl peptidase-4. NSAIDs = non-steroidal anti-inflammatory drugs. PIMs = potentially inappropriate medications.PPIs $=$ proton pump inhibitors. TZDs $=$ thiazolidinediones

The two subgroups did not differ significantly in terms of overall biological parameters, except for the potassium level, which was lower in the PIMs subgroup (4.42 \pm 0.55 versus $4.59 \pm 0.54 \mathrm{mmol} / \mathrm{L} ; P$ $=0.021$; data not shown).

Table 2 shows the medications used by all patients and a comparison between patients with and without PIMs. Patients in the PIMs subgroup were more frequently treated with $\mathrm{CCBs}(P=0.033)$, ACE inhibitors $(P=0.006)$, PPls $(P<0.001)$, aspirin $(P<0.001)$, statins $(P<0.001)$, and antihistamines $(P$ $=0.007$ ).

Among patients with PIMs, 58 patients received diuretics; one methyldopa; one alpha-blockers; one corticosteroids; two hydroxychloroquine; two sulfasalazine; one betahistine; two methotrexate; seven mebeverine; two antiemetics; two tricyclic antidepressants; seven anticonvulsants; four vasodilators; two serotonin-norepinephrine reuptake inhibitors; one memantine; two levodopa; one muscle relaxant; one rivaroxaban; three isosorbide dinitrate; and seven allopurinol among patients without PIM use. There was no documented use of these medications among patients without PIMs. By contrast, one patient used tolterodine in the PIMs subgroup versus no patients in the subgroup without PIMs (data not shown).

Concerning the frequencies of the most common PIMs in the population study as per the Beers criteria, $39.4 \%$ were related to PPIs, $25.2 \%$ to diuretics (other than spironolactone), $10.6 \%$ to NSAIDs, and $8.7 \%$ to aspirin. 


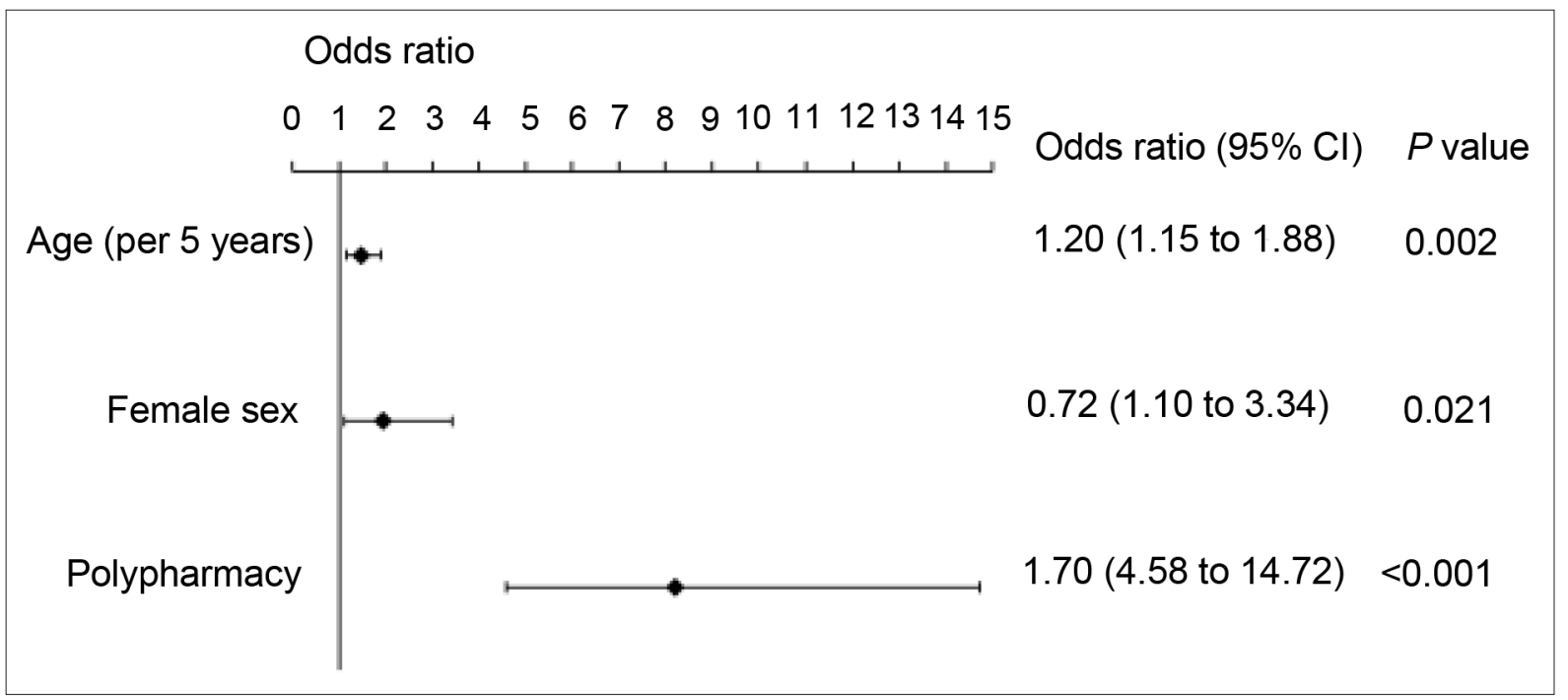

Figure 1 Analysis of factors associated with potentially inappropriate medication using a multiple logistic regression model

In order to identify independent factors for PIMs, analyses were conducted using multiple logistic regression (Figure 1), including and controlling for all variables, revealing differences under univariate analysis. The multivariate analysis identified three independent parameters associated with PIMs: incremental age per 5 years $(r=0.39 \pm 0.12 ; P=0.002)$, female sex $(r=0.66 \pm 0.29 ; P=0.021)$, and polypharmacy $(2.11 \pm 0.29 ; P<0.001)$. The model accounted for $46 \%$ of the total variance in PIMs.

\section{Discussion}

\section{Summary}

This study showed a highly prevalent use of PIMs $(60.7 \%, n=164)$. Incremental age per 5 years, female sex, and polypharmacy were identified as independent parameters associated with PIMs. Oral antidiabetic agents (long-acting sulfonylureas), PPIs, diuretics, NSAIDs, and aspirin were the most commonly used PIMs.

\section{Strengths and limitations}

Although the Saudi population is a growing one, with a projected increased life expectancy ${ }^{11}$ and high prevalence of chronic diseases, there are few studies concerning the use of PIMs in this population. The strength of the present study, compared to the currently available data from retrospective studies, is that it was conducted prospectively in a large general practice setting.

One limitation of the study was that the authors were not able to verify adherence to medications. Without this limitation, the authors are confident that the present study would have provided a better insight into the use of PIMs in general practice in Saudi Arabia.

Excluding hypertension and osteoarthritis, the frequency of morbidities was not significantly different among patients with PIMs compared to those without PIMs; any interpretation in this regard should be made with caution given the small subgroup sizes, which may have led to an underpowered statistical comparison. The present study had no missing data, except for some blood analysis.

\section{Comparison with existing literature}

The rate of PIMs $(60.7 \%, n=164)$ is higher than the rates previously reported in the US, Canada, and Italy, and even higher than previous studies in Saudi Arabia that reported high rates of PIM use. ${ }^{7,12,14,18}$ Previous studies conducted in Saudi Arabia showed PIMs rates of $52.5 \%$ and $2.1 \%-2.5 \%$. ${ }^{19}$ Differences in the tested populations of these studies may have contributed to the discrepancy between the reported results from previous studies and those obtained herein, since the present study was conducted in a family medicine setting; in Al Odhayani et al, 798 older patients were arbitrarily selected from Prince Sultan Medical Military City through the patient register from the 
family medicine chronic disease clinics in the Home Health Care programme; in Al-Omar et al, ${ }^{19}$ the authors conducted a retrospective cross-sectional study of outpatient pharmacy prescription records of older patients at Riyadh Military Hospital.

In 2017, the general authority for statistics in Saudi Arabia conducted the Elderly Survey, ${ }^{20}$ showing that $4.19 \%$ of the total Saudi population are aged $\geq 65$ years, with the female population representing $51.1 \%$ of the older population. This older population survey showed high prevalence of hypertension (28.5\%) and diabetes mellitus $(28.7 \%)$ among the older Saudi population. A retrospective crosssectional study ${ }^{21}$ targeting 3009 older Saudi patient profiles revealed that $55 \%$ were suffering from polypharmacy, with an average of 6.4 medications prescribed for patients aged 65-70 years, and an average of 4.2 medications prescribed for those aged $\geq 71$ years.

The present study identified incremental age per 5 years, female sex, and polypharmacy as independent parameters associated with PIMs (Figure 1). These findings are similar to what has been reported in previous studies. In a large study conducted in an outpatient setting, female sex and polypharmacy were significantly associated with PIM prescribing. ${ }^{22}$ A further study showed that PIM use increased considerably with older age. ${ }^{23}$

Previous studies have reported that polypharmacy was associated with poor health outcomes, particularly when used by the older patient population. ${ }^{4,10}$ Findings in the present study are consistent with previous literature that identified polypharmacy as a risk factor for PIM use. ${ }^{424-26}$ This is particularly true among the older population when these patients were identified to have received PIMs in the emergency room, where patients come with multiple morbidities. ${ }^{24-26}$ Because of this, the treating physician, together with the pharmacist, is in the perfect position to identify patients who are potential users of PIMs and provide evidence-based guidelines and recommendations to circumvent overuse of medications.

The present study highlights that female sex, independent of polypharmacy, was a significant risk factor for PIMs. These results are supported by previous studies that showed polypharmacy and female sex to be important determinants for an increased likelihood of receiving a PIM prescription. ${ }^{27-29}$

Findings in the present study confirm the high prevalence of both hypertension and diabetes among the older Saudi population ( $72.6 \%$ and $54.1 \%$, respectively). Such a high prevalence of hypertension and diabetes was anticipated because the enrolment was not from the community (that is, healthy population). The FCM clinics are based in a medical city (a tertiary care institute) and receive patients referred from PHCs. Supriya et al reported that older hypertensive patients encountered more PIMs; ${ }^{30}$ however, neither hypertension nor anti-hypertensive drugs were identified as independent factors associated with PIMs in the multivariate logistic regression model (Figure 1).

The present study showed that oral antidiabetic agents (long-acting sulfonylureas), PPIs, diuretics, NSAIDs, and aspirin are the most commonly used PIMs. The other recorded PIMs, with lower use frequencies, were hydralazine, ranitidine, chlorpheniramine, amitriptyline, nifidipine, methyldopa, metoclopramide, pergabaline, and prazosin. The strength of recommendation was strong for all of the above PIMs excluding hydralazine, which was weak following Beers criteria.

The Beers criteria recommend avoiding the use of oral long-acting sulfonylureas (glyburide) since its inappropriate use in older adults was found to be significantly associated with a high risk of severe prolonged hypoglycaemia. ${ }^{9,31}$

As mentioned in the results section, all 58 patients who used diuretics were included in the PIMs group. Beers criteria recommend caution with their use, and stress the need to monitor sodium level. ${ }^{9}$ Diuretics are usually the recommended first-line antihypertensive drug for older patients; however, the risk of developing hyponatraemia in older patients is high, particularly among females (OR 3.10), compared to younger patients with hypertension. ${ }^{32}$ Dehydration and electrolyte disturbances also occur as a result of overuse or inappropriate use of diuretics. ${ }^{33}$

In the present study, 86 patients from the 94 treated by PPIs were recorded as using PIMs since Beers criteria recommended avoiding PPI use for $>8$ weeks unless a clear indication exists. ${ }^{9}$ Indeed, long-term or inappropriate use of PPIs may lead to severe hypochlorhydria, which leads to bacterial colonisation and increased susceptibility to infection among older patients. ${ }^{34}$

Due to a lack of evidence of benefit versus risk in adults aged $\geq 80$ years, Beers criteria recommend using aspirin with caution in this age group. ${ }^{9}$ Therefore, 16 patients prescribed aspirin for primary prevention of cardiac events were identified as PIMs in the present study, from 112 total aspirin prescriptions. 
The recommendation of Beers criteria regarding the use of NSAIDs is 'to avoid chronic use unless other alternatives are not effective and the patient can take gastroprotective agent (PPI or misoprostol)'. ${ }^{9}$ This recommendation allowed the authors to identify 23 NSAIDs prescriptions as PIMs among the total of 55 taken by the study population. NSAIDs represented $10.6 \%$ of the total PIMs. A similar rate was reported by Osei et al. ${ }^{8}$

\section{Implications for research and practice}

The present study reinforces the importance of routine medication reviews, especially in older patients. It will be useful to integrate the Beers criteria in the health informatics system as part of Clinical Decision Support to alert physicians and pharmacists to avoid any potential harm related to PIMs. The widespread dissemination of the criteria in education and training to all levels of healthcare practitioners should also take place.

The impact of this research could be significant in terms of supporting local and regional knowledge of PIMs and polypharmacy in the older patient population. Moreover, this study could pave the way for further investigations to validate the present study findings on a larger population in different settings, including those patients previously mentioned that were seen at the emergency room. Future research should evaluate interventions aimed at improving primary care follow-up and reducing the use of PIMs.

The present study showed high prevalence of PIMs, following Beers criteria, among older patients followed-up in FCM clinics in Saudi Arabia. Incremental age per 5 years, female sex, and polypharmacy were found to be associated with PIM use. The frequency of morbidities was not significantly different among patients with PIMs compared to those without PIMs, except for hypertension and osteoarthritis, which were more common in patients with PIMs than without. Long-acting sulfonylureas, PPIs, diuretics, NSAIDs, and aspirin were the most common PIMs. The present study reinforces the importance of routine medication reviews, especially in the older patient population.

\section{Funding}

N/A

\section{Ethical approval}

The present study was approved by the Ethical and Institutional Review Board of KSMC prior to the conduct of the study (reference number: H1R1-14-Jul17-01).

Provenance

Freely submitted; externally peer reviewed

Acknowledgements

The authors are grateful to Family Medicine Academy of KSMC for supporting this study.

\section{References}

1. Aparasu RR, Mort JR. Inappropriate prescribing for the elderly: Beers criteria-based review. Ann Pharmacother 2000; 34(3): 338-346. DOI: https://doi.org/10.1345/aph.19006

2. Lau DT, Kasper JD, Potter DEB, et al. Hospitalization and death associated with potentially inappropriate medication prescriptions among elderly nursing home residents. Arch Intern Med 2005; 165(1): 68-74. DOI: https://doi.org/10.1001/archinte.165.1.68

3. Trygstad TK, Christensen D, Garmise J, et al. Pharmacist response to alerts generated from Medicaid pharmacy claims in a long-term care setting: results from the North Carolina polypharmacy initiative. J Manag Care Pharm 2005; 11(7): 575-583. DOI: https://doi.org/10.18553/jmcp.2005.11.7.575

4. Frazier SC. Health outcomes and polypharmacy in elderly individuals: an integrated literature review. J Gerontol Nurs 2005; 31(9): 4-11. DOI: https://doi.org/10.3928/0098-9134-20050901-04

5. Lim Y-J, Kim H-Y, Choi J, et al. Potentially inappropriate medications by Beers criteria in older outpatients: prevalence and risk factors. Korean J Fam Med 2016; 37(6): 329-333. DOI: https://doi.org/10.4082/kifm.2016.37.6. 329

6. Henschel F, Redaelli M, Siegel M, Stock S. Correlation of incident potentially inappropriate medication prescriptions and hospitalization: an analysis based on the PRISCUS list. Drugs Real World Outcomes 2015; 2(3): 249-259. DOI: https://doi.org/10.1007/s40801-015-0035-4 
7. Al Odhayani A, Tourkmani A, Alshehri M, et al. Potentially inappropriate medications prescribed for elderly patients through family physicians. Saudi J Biol Sci 2017; 24(1): 200-207. DOI: https://doi.org/10.1016/j.sjbs.2016.05.006

8. Osei EK, Berry-Cabán CS, Haley CL, Rhodes-Pope H. Prevalence of beers criteria medications among elderly patients in a military Hospital. Gerontol Geriatr Med 2016; 2: 233372141663779. DOI: https://doi.org/10.1177/ 2333721416637790

9. The American Geriatrics Society 2015 Beers Criteria Update Expert Panel. American Geriatrics Society 2015 updated Beers criteria for potentially inappropriate medication use in older adults. J Am Geriatr Soc 2015; 63(11): 2227-2246. DOI: https://doi.org/10.1111/jgs.13702

10. PattersonSM, HughesC, KerseN, et al. Interventions to improve the appropriate use of polypharmacy for older people. Cochrane Database Syst Rev 2012; 5: CD008165. DOI: https://doi.org/10.1002/14651858.CD008165.pub2

11. United Nations Economic and Social Commission for Western Asia. The demographic profile of Saudi Arabia. 2017; https://www.unescwa.org/sites/www.unescwa.org/files/saudi_arabia_2017-single_pages_jan_5.pdf (accessed 8 Jan 2020).

12. Morgan SG, Hunt J, Rioux J, et al. Frequency and cost of potentially inappropriate prescribing for older adults: a cross-sectional study. CMAJ Open 2016; 4(2): E346-E351. DOI: https://doi.org/10.9778/cmajo.20150131

13. Voigt $\mathrm{K}$, Gottschall $\mathrm{M}$, Köberlein-Neu J, et al. Why do family doctors prescribe potentially inappropriate medication to elderly patients? BMC Fam Pract 2016; 17: 93. DOI: https://doi.org/10.1186/s12875-016-0482-3

14. Amos TB, Keith SW, Del Canale S, et al. Inappropriate prescribing in a large community-dwelling older population: a focus on prevalence and how it relates to patient and physician characteristics. J Clin Pharm Ther 2015; 40(1): 7-13. DOI: https://doi.org/10.1111/jcpt.12212

15. Chang C-B, Lai H-Y, Yang S-Y, et al. Patient- and clinic visit-related factors associated with potentially inappropriate medication use among older home healthcare service recipients. PLoS One 2014; 9(4): e94350. DOI: https://doi.org/10.1371/journal.pone.0094350

16. Husson N, Watfa G, Laurain M-C, et al. Characteristics of polymedicated ( $\geq 4$ ) elderly: a survey in a communitydwelling population aged 60 years and over. J Nutr Health Aging 2014; 18(1): 87-91. DOI: https://doi.org/10.1007/ s12603-013-0337-8

17. Metcalfe C. Biostatistics: a foundation for analysis in the health sciences. 7th EDN. Wayne W. Daniel, Wiley, 1999. No. of. Pages: xiv+755+appendices. price: 28.95. ISBN 0-471-16386-4. Stat Med 2001; 20(2): 324-326. DOI: https://doi.org/10.1002/1097-0258(20010130)20:2<324::AID-SIM635>3.0.CO;2-O

18. Berdot S, Bertrand M, Dartigues J-F, et al. Inappropriate medication use and risk of falls - a prospective study in a large community-dwelling elderly cohort. BMC Geriatr 2009; 9: 30. DOI: https://doi.org/10.1186/1471-2318-9-30

19. Al-Omar HA, Al-Sultan MS, Abu-Auda HS. Prescribing of potentially inappropriate medications among the elderly population in an ambulatory care setting in a Saudi military hospital: trend and cost. Geriatr Gerontol Int 2013; 13(3): 616-621. DOI: https://doi.org/10.1111/j.1447-0594.2012.00951.x

20. General Authority for Statistics. Elderly survey. 2017; https://www.stats.gov.sa/sites/default/files/elderly_survey_ 2017en.pdf (accessed 8 Jan 2020).

21. Alsuwaidan A, Almedlej N, Alsabti S, et al. A comprehensive overview of polypharmacy in elderly patients in Saudi Arabia. Geriatrics 2019; 4(2): 3615 May 2019. DOl: https://doi.org/10.3390/geriatrics4020036

22. Buck MD, Atreja A, Brunker $\mathrm{CP}$, et al. Potentially inappropriate medication prescribing in outpatient practices: prevalence and patient characteristics based on electronic health records. Am J Geriatr Pharmacother 2009; 7(2): 84-92. DOI: https://doi.org/10.1016/j.amjopharm.2009.03.001

23. Mo L, Ding D, Pu S-Y, et al. Patients aged 80 years or older are encountered more potentially inappropriate medication use. Chin Med J 2016; 129(1): 22-27. DOI: https://doi.org/10.4103/0366-6999.172558

24. Hohl CM, Dankoff J, Colacone A, Afilalo M. Polypharmacy, adverse drug-related events, and potential adverse drug interactions in elderly patients presenting to an emergency department. Ann Emerg Med 2001; 38(6): 666-671. DOI: https://doi.org/10.1067/mem.2001.119456

25. Bushardt RL, Massey EB, Simpson TW, et al. Polypharmacy: misleading, but manageable. Clin Interv Aging 2008; 3(2): 383-389. DOI: https://doi.org/10.2147/CIA.S2468

26. Banerjee A, Mbamalu D, Ebrahimi S, et al. The prevalence of polypharmacy in elderly attenders to an emergency department — a problem with a need for an effective solution. Int J Emerg Med 2011; 4(1): 22. DOI: https://doi. org/10.1186/1865-1380-4-22

27. Oliveira MG, Amorim WW, de Jesus SR, et al. Factors associated with potentially inappropriate medication use by the elderly in the Brazilian primary care setting. Int J Clin Pharm 2012; 34(4): 626-632. DOI: https://doi.org/10. 1007/s11096-012-9656-9

28. Klarin I, Wimo A, Fastbom J. The association of inappropriate drug use with hospitalisation and mortality: a population-based study of the very old. Drugs Aging 2005; 22(1): 69-82. DOI: https://doi.org/10.2165/00002512200522010-00005

29. Goltz L, Kullak-Ublick GA, Kirch W. Potentially inappropriate prescribing for elderly outpatients in Germany: a retrospective claims data analysis. Int J Clin Pharmacol Ther 2012; 50(3): 185-194. DOI: https://doi.org/10.5414/ CP201441

30. SupriyaKH, Shashi KumarNS, PrakashGM, SareethaA. A prospective observational study of prescription appropriateness of elderly hypertensive patients using Beers criteria in a tertiary care teaching hospital. International Journal of Basic \& Clinical Pharmacology 2017; 6(11): 2545-2550.

31. Shorr RI, Ray WA, Daugherty JR, Griffin MR. Individual sulfonylureas and serious hypoglycemia in older people. J Am Geriatr Soc 1996; 44(7): 751-755. DOI: https://doi.org/10.1111/j.1532-5415.1996.tb03729.x

32. Sharabi $Y$, Illan $R$, Kamari $Y$, et al. Diuretic induced hyponatraemia in elderly hypertensive women. J Hum Hypertens 2002; 16(9): 631-635. DOI: https://doi.org/10.1038/sj.jhh.1001458 
33. Cumming K, Hoyle GE, Hutchison JD, Soiza RL. Prevalence, incidence and etiology of hyponatremia in elderly patients with fragility fractures. PLoS One 2014; 9(2): e88272. DOI: https://doi.org/10.1371/journal.pone.0088272

34. Bavishi C, Dupont HL. Systematic review: the use of proton pump inhibitors and increased susceptibility to enteric infection. Aliment Pharmacol Ther 2011; 34(11-12): 1269-1281. DOI: https://doi.org/10.1111/j.1365-2036.2011. 04874.x 